\title{
Uma revisão do perfil clínico-epidemiológico e das repercussões perinatais em portadoras de síndrome hipertensiva gestacional
}

\author{
A review of the clinical and demographic characteristics and perinatal outcomes \\ in women with gestational hypertension syndrome
}

\author{
Fernanda Miranda Morais* \\ Juliana Mendes Novaes* \\ Maria Clara Amaral Silveira* \\ Mirtz Janiny Alves Rodrigues Araujo* \\ Sâmia Machado Melo* \\ Lucília Silva Gontijo**
}

Resumo: Sabendo que a literatura é sempre agregadora e tendo em vista a multiplicidade de fatores predisponentes ao desenvolvimento da Síndrome Hipertensiva Gestacional (SHG), se solidifica como principal objetivo deste estudo, traçar uma revisão bibliográfica sobre o perfil clínico-epidemiológico de gestantes de alto risco portadoras de SHG, bem como suas repercussões perinatais. A SHG perfaz-se por doença incurável, salvo pela interrupção da gravidez. Ocupa a terceira causa de morte materna no mundo e a principal causa de morte materna no Brasil, atribuindo-se tais índices à grande quantidade de complicações. Pronuncia-se então, a necessidade de identificação precoce destas gestações, com o estudo complementar do ambiente fetal por meio de instrumentos que possibilitam avaliar a presença de hipóxia e suas conseqüências, sendo desejável a regulagem dos níveis pressóricos dentro dos parâmetros seguros para mãe e feto, cooperando deste modo para a redução da morbimortalidade neonatal. Sendo assim, este trabalho intenta agregar informação para que, cada vez mais, os profissionais da saúde permaneçam atentos aos vários aspectos de apresentação deste evento e assim estejam aptos na minimização dos efeitos advindos. Todo o arsenal de avaliações/ferramentas deve ser somado, para delinear a propedêutica, identificar e contornar tais vicissitudes além da decisão entre manutenção/interrupção da gravidez. Com base nesses achados, considera-se primordial uma assistência pré-natal de qualidade, em que as gestantes de risco sejam identificadas desde a anamnese até o exame clínico.

Palavras-chave: Hipertensão na gravidez, síndrome HELP, doença hipertensiva específica da gravidez, pré-eclâmpsia.

\begin{abstract}
Knowing that literature is always aggregator and in view of the multiplicity of factors predisposing to the development of Gestational Hypertension Syndrome (GHS), solidifies as the primary study objective, draw a literature review on the clinical and epidemiological profile of high-risk pregnancies carriers SHG and their perinatal outcomes. The GHS makes up for incurable except by termination of pregnancy. Occupies the third leading cause of maternal death worldwide and the leading cause of maternal death in Brazil, attributing such indices to lots of complications. Pronounced then the need for early identification of these pregnancies, with additional study of the fetal environment through tools that enable the evaluation of the presence of hypoxia and its consequences, and it is desirable to regulate blood pressure levels within the parameters safe for mother and fetus, thus cooperating to reduce neonatal mortality.

\footnotetext{
* Acadêmica do Curso de Graduação em Medicina das Faculdades Integradas Pitágoras.

* Acadêmica do Curso de Graduação em Medicina das Faculdades Integradas Pitágoras.

* Acadêmica do Curso de Graduação em Medicina das Faculdades Integradas Pitágoras.

* Acadêmica do Curso de Graduação em Medicina das Faculdades Integradas Pitágoras.

* Acadêmica do Curso de Graduação em Medicina das Faculdades Integradas Pitágoras.

** Docente das FIPMoc e Mestre em Farmacologia Terapêutica e Experimental da Universidade Federal do Rio de Janeiro - UFRJ.
} 
Thus, this paper attempts to aggregate information so that, increasingly, health professionals remain aware of various aspects of presenting this event and so are able to minimize the effects arising. The whole arsenal of assessments / tools should be added to delineate the workup, identify and overcome such vicissitudes beyond the decision between maintenance/termination of pregnancy. Based on these findings, it is considered one primary prenatal care quality in pregnant women at risk are identified from the interview to the clinical examination.

Keywords: Hypertension in pregnancy, HELLP syndrome, gestational hypertension syndrome, pre-eclampsia.

\section{Introdução}

A expressão "hipertensão na gravidez" recebe a designação geral de síndromes hipertensivas gestacionais (SHG). Estas são caracterizadas por níveis pressóricos iguais ou acima de $140 \mathrm{mmHg}$ para a pressão sistólica e $90 \mathrm{mmHg}$ para pressão diastólica (ASSIS, 2008). É uma doença incurável, exceto pela interrupção da gravidez, e pode evoluir para quadros ainda mais complexos, como eclâmpsia, síndrome HELLP (haemolysis, elevated liver enzyme activity e low platelets) ou CID (coagulação intravascular disseminada) (DUSSE et al., 2001).

Para o consenso do National High Blood Pressure Education Program (NHBPEP), publicado em 1990 e reafirmado em 2000, na identificação das formas de manifestação da hipertensão arterial na gravidez é fundamental diferenciar a hipertensão que antecede a gravidez, daquela que é condição específica da mesma. Na primeira, a elevação da pressão arterial é o aspecto fisiopatológico básico da doença, a segunda é resultado da má adaptação do organismo materno à gravidez, sendo a hipertensão apenas um de seus achados (PERAÇOLI; PARPINELLI, 2005).

As síndromes hipertensivas da gravidez, nos países desenvolvidos, ocorrem entre $2 \%$ e $8 \%$ das gestações, podendo, no Brasil, chegar a $30 \%$, representando a terceira causa de morte materna no mundo e a principal causa de morte materna no Brasil. Estes altos índices de mortalidade são devidos a uma grande quantidade de complicações, como a progressão da hipertensão, a pré-eclâmpsia superposta, eclâmpsia, descolamento prematuro de placenta normoinserida, síndrome HELLP, tromboembolismo, edema pulmonar, hemorragia cerebral, encefalopatia, CID, insuficiência renal e hepática (SOUZA et al., 2010).

A incidência da SHG é apresentada, como sendo merecedora de maiores investigações, tendo em vista a multiplicidade de fatores que podem predispor a mulher gestante a desenvolver a doença. Entre eles destacam-se: paridade; gemelaridade; nível sócio-econômico, principalmente no que se refere ao acesso aos serviços de saúde; estado nutricional; entre outros (GONÇALVES et al., 2005).

Considerando a importância da gravidez de alto risco no binômio saúde-doença materno-fetal, é necessária a identificação precoce destas gestações, para que assim seja possível proporcionar uma assistência médica adequada e obter resultados satisfatórios, uma vez que, os óbitos decorrentes das complicações da gravidez são, em sua maioria, preveníveis. 
Para tanto, é preciso investigar as características individuais e condições sóciodemográficas das grávidas, bem como a história reprodutiva anterior à gestação atual, as doenças obstétricas e as intercorrências clínicas. A escassez de estudos regionais atualizados sobre o assunto torna necessária a aquisição de novos dados acerca da gravidez de alto risco, no sentido de manter os profissionais da área de saúde sempre atentos aos vários aspectos de apresentação deste evento, para que assim possam proceder corretamente minimizando os efeitos adversos sobre a mãe e o filho.

Diante de tais implicações, é que se embasa a curiosidade de se explanar acerca das repercussões oriundas da síndrome hipertensiva gestacional. Cônscios de que o tratamento definitivo da pré-eclâmpsia é o parto, e que na dependência de diversos fatores a interrupção da gravidez estará ou não indicada; e de que a instalação precoce da doença aumenta a chance de prematuridade com subseqüente incremento da morbimortalidade perinatal; e ainda, sabedores de que várias condutas têm sido propostas enquanto não é possível ou recomendável interromper tal gestação, goza-se de razão plausível, para uma assistência pré-natal qualificada com profissionais gabaritados para tal cuidado e com 0 instrumental suficiente e eficiente para identificar e contornar tais vicissitudes, na tentativa de prevenir, as já ditas, complicações perinatais.

Neste sentido, os cuidados especializados por parte dos profissionais envolvidos no atendimento dessas gestantes de risco, podem evitar complicações graves. Desta forma, o estudo objetiva-se traçar uma revisão bibliográfica sobre o perfil clínico-epidemiológico de gestantes de alto risco portadoras de síndrome hipertensiva gestacional, bem como suas repercussões perinatais.

\section{Procedimentos Metodológicos}

Esse trabalho trata-se de uma pesquisa de revisão interativa de literatura, devido a sua característica predominante que é um amplo levantamento bibliográfico. Pelo seu delinemaneto metodológico do estudo, procedeu-se com coleta de dados a partir de fontes secundárias.

Para acesso aos artigos que compuseram a amostra da presente revisão utilizouse a base de dados da Biblioteca Virtual de Saúde (BVS) que organiza a informação em uma estrutura que integra e interconecta várias bases de dados referenciais, como SciELO (Scientific Electronic Library Online), LILACS (Literatura Latino-Americana e do Caribe em Ciências da Saúde), MEDLINE (Literatura Internacional em Ciências da Saúde), consultados via online, utilizando como descritores: complicações do puerpério, hipertensão, síndrome de Hellp, eclâmpsia, pré-eclâmpsia, hipertensão induzida pela gestação e puerpério.

Como critérios de seleção foram incluídos todos os artigos que abordaram as palavras-chaves citadas desde que estivessem disponíveis na íntegra, que versassem sobre complicações decorrentes das síndromes hipertensivas e publicados no período entre 1986 a 2012; sendo a amostra constituída de 31 citações, sendo 27 artigos, 2 livros, 1 dissertação e 1 relatório. 


\section{Síndrome hipertensiva gestacional: aspectos gerais}

Mesmo com os avanços na assistência obstétrica no último século, particularmente no campo da tecnologia, a morbimortalidade materna ainda acompanha a gravidez, parto e puerpério. No Brasil, a morte materna se configura como um problema de saúde pública e, segundo dados do Ministério da Saúde, em 2006, a razão de mortalidade materna (RMM) foi estimada em $77,2 / 100.000$ nascidos vivos (NV). As regiões Norte e Nordeste têm os piores indicadores, enquanto as regiões Sul e Sudeste têm as menores RMM (MORSE et al., 2011).

A Organização Mundial da Saúde (OMS) considera até 20 mortes por 100 mil NV índices aceitáveis de mortalidade materna, além disso, classifica a mortalidade materna como baixa (até 20), média (20 a 49), alta (50 a 149) e muito alta (acima de 150/100 mil NV) (ORGANIZAÇÃO MUNDIAL DA SAÚDE, 2012). Portanto, considerando a classificação da OMS nossos índices continuam altos.

As causas obstétricas diretas são responsáveis por $75 \%$ dos óbitos maternos, sendo as síndromes hipertensivas as principais causadoras, seguidas de hemorragias, e infecções puerperais (MORSE et al., 2011).

Em conformidade, Novo; Gianini (2010) mostraram em seus relatos que mesmo com o decorrer dos anos a hipertensão é a principal complicação da gravidez e de morte materna e consideram ainda que a má qualidade na assistência tanto no pré-natal quanto no ambiente hospitalar durante e após o parto é um dos determinantes para este índice. De fato, espera-se que, quanto melhor equipado e estruturado o serviço de saúde de um determinado local, menor sejam seus índices de mortalidade materna e fetal, no qual a gestante é orientada e esclarecida em relação à gestação e as possíveis complicações desse período.

As síndromes hipertensivas gestacionais consistem no resultado da má adaptação do organismo materno a gravidez, classificando-se em: Hipertensão gestacional, presença de hipertensão arterial transitória na gravidez, sem proteinúria e normalização da pressão arterial após a $12^{a}$ semana de gestação; Pré-eclâmpsia, forma não convulsiva marcado pelo início da hipertensão aguda após a vigésima semana de gestação e Eclâmpsia, distúrbio hipertensivo gestacional que se caracteriza pelos episódios convulsivos (ANGONESI; POLATO, 2007).

Em 20-50\% das pacientes com hipertensão gestacional há progressão para préeclâmpsia, ou seja, há desenvolvimento de proteinúria, podendo a mesma se desenvolver antes ou após o parto (OLIVEIRA et al., 2006). A eclâmpsia pode se apresentar de forma isolada ou associada à hipertensão arterial materna preexistente, sendo a forma mais grave dos distúrbios hipertensivos (NOVO; GIANINI, 2010).

Dentre as diversas complicações das síndromes hipertensivas encontra-se a síndrome de HELPP caracterizada por: $(H)$ hemólise, (EL) enzimas hepáticas elevadas e (LP) baixa contagem de plaquetas. Essa síndrome pode levar a insuficiência cardíaca e pulmonar, insuficiência renal aguda e síndrome da angustia respiratória (ANGONESI; POLATO, 2007). 


\section{Clínica e epidemiologia da síndrome hipertensiva gestacional}

A maioria das gestações transcorre sem intercorrências, caracterizando-se como um período de higidez da mãe e do concepto. Entretanto, parte das gestantes pode apresentar complicações de elevado risco de morbidade e mortalidade materna e fetal, como a SHG. Como já dito, dentre as formas clínicas que esta síndrome pode apresentar destaca-se a pré-eclâmpsia, definida pela presença de hipertensão e de proteinúria após a $20^{a}$ semana de gestação, podendo ser leve ou grave (MOURA et al., 2010).

De acordo com um consenso realizado em 2000 nos EUA (National High Blood Pressure Education Program Working Group on High Blood Pressure in Pregnancy), através de uma combinação de medicina baseada em evidências com a opinião de especialistas, a definição de pré-eclâmpsia foi dada por hipertensão (pressão arterial sistólica maior ou igual que $140 \mathrm{mmHg}$ e/ou diastólica maior ou igual que $90 \mathrm{mmHg}$ ), que surge depois de 20 semanas de gravidez, associada com proteinúria (excreção de proteína na urina) $>300 \mathrm{mg}$ nas 24 horas (NHBPEP, 2000).

Apesar da fisiopatologia da pré-eclampsia ainda ser desconhecida, é amplamente aceito, atualmente, o fato de que a isquemia é um fator primordial. Durante o início do segundo trimestre da gestação (entre a $18^{\mathrm{a}}$ e $20^{\mathrm{a}}$ semana) instala-se um processo referido como "pseudovasculogenese", caracterizado pela migração dos citotrofoblastos em direção às arteríolas uterinas espiraladas onde sofrem diferenciação em células com fenótipo endotelial. Nesse processo, ocorre remodelamento gradual da camada endotelial desses vasos e destruição do tecido elástico-muscular das artérias e arteríolas, tornando-as mais dilatadas.

Essa migração/diferenciação dos citotrofoblastos deve-se a alterações nos perfis de expressão de certas citocinas, moléculas de adesão, constituintes da matriz extracelular, metaloproteinases e o antígeno de histocompatibilidade (HLA-G). O remodelamento das artérias uterinas espiraladas resulta na formação de um sistema local de baixa resistência arteriolar que é essencial ao aumento do suprimento sanguíneo para o desenvolvimento e crescimento do feto. Essa falência do remodelamento vascular impede uma resposta adequada ao aumento da demanda do fluxo sanguíneo que ocorre durante a gestação, diminuindo a perfusão útero-placentária e provocando isquemia da placenta (CAVALLI et al., 2009).

A hipóxia placentária aumenta a produção de radicais livres que lesam o endotélio. Essa lesão, por sua vez, desencadeia a ativação plaquetária, a liberação de tromboxano e serotonina e a agregação plaquetária, com obstrução do fluxo sangüíneo placentário. A hipóxia placentária também aumenta a produção de peróxidos lipídicos que prejudicam a síntese de prostaciclina, potente vasodilatador. A lesão endotelial também pode produzir pró-coagulantes e aumentar a permeabilidade capilar. Por fim, aumento anormal do cálcio livre intracelular e da atividade dos fatores de coagulação, bem como alteração do manuseio dos ácidos gordurosos pelo fígado, também estão implicados na patogênese da pré-eclâmpsia (GANEM; CASTIGLIA, 2002).

A hipertensão arterial decorrente da elevação na resistência vascular é o sinal precoce da pré-eclâmpsia. A maior resistência vascular é resultado do aumento 
da atividade simpática, uma resposta exagerada à adrenalina e à noradrenalina circulantes, e do aumento da sensibilidade à angiotensina II. O edema pulmonar, apesar de raro, é complicação séria da pré-eclâmpsia. A baixa pressão coloidosmótica, o aumento da pressão hidrostática intravascular e o aumento na permeabilidade capilar decorrente da lesão endotelial contribuem para o desenvolvimento desse edema. As convulsões eclâmpticas são as mais sérias complicações da pré-eclâmpsia. Cefaléia, distúrbios visuais, dor epigástrica ou no quadrante superior direito do abdômen são sintomas comuns que antecedem a convulsão. Há lesão endotelial imunorreativa que determina agregação plaquetária e depósito de fibrina no leito vascular renal. A diminuição no fluxo sangüíneo renal e na taxa de filtração glomerular, assim como o aumento concomitante nas concentrações de renina, de fator natriurético atrial, de angiotensina e de catecolaminas pode resultar em oligúria e insuficiência renal. As alterações hipertensivas são responsáveis por $50 \%$ dos casos de insuficiência renal aguda na gestação (GANEM; CASTIGLIA, 2002).

Placenta, rins, fígado e cérebro têm suas funções deprimidas em até $60 \%$, o que comprova que os distúrbios hipertensivos na gravidez são graves, pois intensificam os riscos de descolamento prematuro de placenta, prematuridade, baixo peso ao nascer e óbito materno e fetal (MOURA et al., 2010).

A presença de edemas não faz parte dos critérios diagnósticos, porque não apresenta correlação com o prognóstico gestacional, visto que grávidas perfeitamente normais podem apresentar edemas, mesmo edema generalizado. Exames como hemograma, uréia, creatinina, acido úrico, contagem de plaquetas, teste de função hepática (transaminases) e bilirrubinas fazem parte da propedêutica complementar; mas não como critérios diagnósticos. Estes exames servem para avaliar a gravidade da pré-eclâmpsia, mas o diagnóstico não depende de sua alteração (HERCULANO, 2010).

Embora a água corpórea total esteja aumentada, o volume plasmático está diminuído, observando-se hemoconcentração. A trombocitopenia é a anormalidade hematológica mais freqüente na pré-eclâmpsia, mas raramente é inferior a $100.000 \mathrm{~mm}^{3}$. É decorrente do aumento no consumo de plaquetas (agregação nos locais de lesão endotelial), da diminuição da meia-vida ou da sua destruição por mecanismos imunes. Geralmente, as vias extrínsecas e intrínsecas da coagulação estão normais, demonstrando que a destruição das plaquetas não é provocada pela atividade da trombina e pela formação do coágulo. A coagulação intravascular disseminada é rara e nunca é encontrada na ausência de trombocitopenia moderada ou intensa (GANEM; CASTIGLIA, 2002).

A síndrome HELLP, denominação introduzida à literatura em 1982 por Weinstein, representada por uma tríade laboratorial clássica: $\mathrm{H}$ (hemólise), EL (elevação das enzimas hepáticas) e LP (baixa contagem de plaquetas) se desenvolve como forma atípica e complicada da pré-eclâmpsia grave. Está associada à falência de múltiplos órgãos devido à alteração circulatória generalizada. A incidência varia de 2 a $12 \%$ e é alta em virtude do retardo no seu diagnóstico e na resolução do parto (SILVA et al., 2012).

A literatura diverge em relação aos valores dos parâmetros que definem a síndrome. Assim, a hemólise é definida pela queda no valor do hematócrito abaixo de $38 \%$ e/ou pela dosagem da desidrogenase láctica (LDH) entre $164 \mathrm{U} / \mathrm{L}$ 
e $600 \mathrm{U} / \mathrm{L}$. A elevação de enzimas hepáticas é definida por valor de aspartato aminotransferase sérica (AST) maior que $50 \mathrm{U} / \mathrm{L}$, ou ainda pela elevação de dois ou três desvios-padrão sobre os valores de normalidade estabelecidos, pelos diferentes laboratórios, para o terceiro trimestre da gestação. Sibai et al. (1986), propuseram uma sistematização dos padrões laboratoriais e bioquímicos para o diagnóstico de síndrome HELLP, que foram adotados pelo Ministério da Saúde do Brasil, com o manual de normatização para urgência e emergência obstétrica. A incidência de síndrome HELLP é influenciada pela idade, raça, paridade e duração da pré-eclâmpsia ou presença de eclampsia (PERAÇOLI; PARPINELLI, 2005).

No Brasil, a hipertensão arterial no período gestacional constitui a primeira causa de morte materna. Foi evidenciado recentemente que, nas capitais brasileiras, os transtornos hipertensivos representam em torno de $25 \%$ dos óbitos maternos investigados (LAURENTI et al., 2004). Dados brasileiros demonstram que cerca $10 \%$ das gestações evoluem para o alto risco gravídico e as SHG são as principais causas de óbito materno e fetal (KHAN et al., 2006).

\section{Fatores de risco}

Vários fatores concorrem para o desenvolvimento da síndrome hipertensiva gestacional, sendo a incidência maior quando presente em situações como obesidade, idade nos extremos da fase reprodutiva, diabetes, hipertensão, nefropatias, história familiar ou pessoal de pré-eclâmpsia ou eclâmpsia, dietas hipoprotéicas e hipersódicas, baixa escolaridade e atividade profissional fora do domicílio, grupo sanguíneo $A B$, primigestas, gestações múltiplas, hidropsia fetal e neoplasia trofoblástica. Nesse contexto, o Ministério da Saúde ressalta que a SHG acomete mais as primigestas e mulheres com história pessoal e/ou familiar de pré-eclâmpsia e/ou eclâmpsia, com gestação gemelar, doença cardiovascular pré-existente, hipertensão, nefropatia, lúpus e diabetes, o que corresponde aos principais fatores de risco para a doença (MOURA et al., 2010).

A literatura normalmente descreve a prevalência de pré-eclâmpsia como sendo de $5 \%$ a $8 \%$, apresentando variações. Em gestação gemelar a prevalência de pré-eclâmpsia é de $14 \%$, podendo alcançar $40 \%$ em pacientes com préeclâmpsia prévia (ACOG, 2002).

A idade materna é fator determinante de complicações durante o período gravídico. A gestação de uma jovem, bem como a gestação que ocorre em idade avançada, é considerada de risco gestacional para a pré-eclâmpsia (MOURA et al., 2010).

Em adolescentes primigestas, alguns fatores, como ignorância da própria gravidez, vergonha, gestação indesejada e demora em procurar a assistência pré-natal, podem contribuir para aumentar o risco desta síndrome (COSTA et al., 2003).

Relatos em diferentes estudos mostram que a situação socioeconômica é uma condição de risco para as SHG, uma vez que estas são maiores em países em desenvolvimento do que nos desenvolvidos (OLIVEIRA; FREITAS, 2002). 
O perfil sociodemográfico das gestantes pode ser observado no estudo exploratório descritivo desenvolvido no Núcleo de Atenção Médica Integrada (NAMI), da Universidade de Fortaleza, no qual $91 \%$ das gestantes estavam na faixa etária de 17 a 31 anos; 74\% eram de cor parda; 62\% cursaram até o ensino fundamental e $72 \%$ informaram ter como religião o catolicismo (SANTOS et al., 2009).

Porém, em outro estudo realizado por Assis et al. (2008) as características sociodemográficas não se configuraram como risco para ocorrência de SHG, visto que a maioria das gestantes estudadas não apresentava baixa condição socioeconômica, corroborando com argumento do National High Blood Pressure Education Program de que o nível socioeconômico não predispõe a população à SHG.

A raça não-branca é fator de risco para pré-eclâmpsia, independente de fatores como idade, pré-eclâmpsia prévia, obesidade e paridade. Possivelmente este fator pode estar associado ao fato da hipertensão arterial ser mais prevalente nesta população (ASSIS et al., 2008).

Diante da hipótese de que o estado emocional interfere na variabilidade da pressão arterial, o estudo de Santos et al. (2009), mostrou ocorrência de conflitos emocionais em pacientes com SHG, sendo estes geralmente associados à recusa da gravidez pela família, pelo parceiro e até pela própria gestante.

A obesidade, bem como a hipertensão arterial crônica (HAC), são consideradas fatores de risco para SHG, sendo um fator preocupante, já que atualmente estas são problemas de saúde pública crescente, contribuindo com o aumento da SHG (ASSIS et al., 2008). A HAC acomete em torno de 5\% das gestações. A taxa de pré-eclâmpsia sobreposta em pacientes hipertensas crônicas é 15 a 25\% (ACOG, 2001).

\section{Repercussões perinatais}

As síndromes hipertensivas também apresentam elevada taxa de morbimortalidade perinatal, oscilando entre $5 \%$ e $20 \%$, devido principalmente à insuficiência uteroplacentária, que ocasiona restrição de crescimento intra-útero (RCIU) e as complicações secundárias a prematuridade. A hipertensão leve encontra-se associada a $33 \%$ de partos prematuros e a $11 \%$ de neonatos pequenos para a idade gestacional (PIG). Enquanto que a hipertensão grave tem sido associada de 62 a $70 \%$ de partos prematuros e a $40 \%$ de neonatos PIGs (BUCHBINDER et al., 2002; SOUZA et al., 2010).

É consenso entre a literatura vigente que a SHG encontra-se entre as principais causas de morte materna e elevada taxa de morbimortalidade perinatal. Na SHG é observada a insuficiência útero-placentária com repercussões cruciais sobre o concepto. Entre as complicações fetais destacam crescimento restrito, prematuridade e mortalidade fetal e neonatal (PASCOAL, 2002).

Acrescentam-se às complicações perinatais supracitadas dos neonatos de mães afetadas por hipertensão gestacional ou crônica, o risco aumentado para recémnascidos pequenos para a idade gestacional, Apgar baixo no primeiro e quinto 
minuto (índice de fatores característicos, tais como, cor, respiração, frequência cardíaca, tônus muscular e reflexos), infecção neonatal, síndrome da aspiração de mecônio e síndrome da angústia respiratória (OLIVEIRA et al., 2006).

As complicações materno-fetais estão relacionadas principalmente aos altos níveis de pressão arterial, à hipertensão mal controlada e ao incremento de comorbidades e/ou fatores de risco. A prematuridade, que é muitas vezes induzida, como única forma de minimizar o agravamento materno ou resolver a insuficiência placentária, contribui para taxas elevadas de mortalidade neonatal, bem como determina sequelas a curto e longo prazo entre os recém-nascidos sobreviventes (CORRÊA et al., 2011).

Dentre as SHG, a pré-eclâmpsia grave é potencialmente a que apresenta pior prognóstico materno-fetal. Conceptos de mães com pré-eclâmpsia ou préeclâmpsia sobreposta têm maiores riscos de prematuridade, ocorrência de partos de fetos pequenos para a idade gestacional, necessidade de Unidade de Tratamento Intensivo (UTI) neonatal, necessidade de suporte ventilatório e maior incidência de mortalidade perinatal, quando comparados aos conceptos de mães portadoras de hipertensão gestacional leve, HAC isolada e normotensas. Apresentam, também, maior ocorrência de doenças associadas à prematuridade, como: síndrome da angústia respiratória, hemorragia intraventricular e enterocolite necrotizante. A piora do prognóstico materno-fetal está diretamente relacionada à gravidade da hipertensão gestacional/pré-eclâmpsia (OLIVEIRA et al., 2006).

O estudo da qualidade do ambiente fetal é feito com vários instrumentos que possibilitam avaliar com razoável eficiência a presença de hipóxia e suas conseqüências, contribuindo para melhorar o prognóstico perinatal. Todas as formas de avaliação devem ser somadas para adequada avaliação da evolução fetal, fazendo parte uma avaliação clínica apurada com medida da altura uterina, palpação do líquido, avaliação quantitativa dos movimentos fetais, e a ausculta dos batimentos cardíacos e associação aos métodos biofísicos: ultrasonografia, cardiotocografia, perfil biofísico fetal e Doppler. Constituindo elementos indispensáveis para a decisão clínica diante da opção entre a manutenção do feto em ambiente hostil e os agravos da prematuridade (SASS, 2007).

A hipertensão leve encontra-se associada a 33\% de partos prematuros e a $11 \%$ de neonatos pequenos para a idade gestacional. Enquanto que a hipertensão grave tem sido associada de 62 a $70 \%$ de partos prematuros e a $40 \%$ de neonatos PIGs (SOUZA et al., 2010).

Outros estudos enfatizam que essas síndromes ocorrem em $6 \%$ a $8 \%$ das gestações, evoluindo, com relativa frequência, a agravos materno-fetais devido à hipóxia intra-uterina. Como alterações tardias, crianças pequenas para a idade gestacional associadas ao diagnóstico de hipertensão gestacional, podem apresentar maiores níveis de pressão arterial e dislipidemia precocemente na fase adulta (FERRÃO et al., 2006; CARVALHO et al., 2008).

Da análise dos 200 partos realizados no Hospital das Clínicas da Universidade Federal do Triângulo Mineiro a média da idade gestacional foi de 37,6 $\pm 3,5$ semanas, sendo $24,5 \%$ dos recém-nascidos considerados prematuros. Em relação ao índice de Apgar, 13,5\% recém-nascidos tiveram índice da Apgar $<7$ no primeiro e no quinto minuto, e a maioria estava associada à pré-eclâmpsia 
materna. Os menores valores para a idade gestacional, peso dos recém-nascidos ao nascimento e para o índice de Apgar no primeiro minuto foram observados nos grupos de pacientes com pré-eclâmpsia e pré-eclâmpsia sobreposta à hipertensão crônica (FERRÃO et al., 2006).

Outros resultados perinatais de gestantes com SHG atendidas na Santa Casa de Misericórdia de Barbacena demonstraram uma taxa de 54,5\% de parto prétermo entre as gestantes hipertensas e de $17,8 \%$ entre as normotensas. Foi confirmado o alto risco de prematuridade para as formas leves e graves da hipertensão, que ocorreu devido ao agravamento do quadro materno e/ou fetal, com registro de taxa de parto pré-termo entre 20 e $40 \%$ chegando a $70 \%$ na hipertensão grave. Notou-se diferença significativa em relação ao baixo peso ao nascimento. Com taxa de $48 \%$ entre as hipertensas e de $13,3 \%$ entre as normotensas podendo ser explicado pela interrupção precoce da gestação devido a agravamento do quadro materno-fetal. Entre as gestantes hipertensas 22,3\% dos recém nascidos foram admitidos em UTI. Sendo que $86,6 \%$ destes ocorreram na forma grave de hipertensão. Também, foi evidenciado no seguinte estudo uma natimortalidade em $4,2 \%$ nas portadoras de SGH (CARVALHO et al., 2008).

\section{Tratamento}

Atualmente existe um grande questionamento quanto ao benefício do tratamento da hipertensão na gravidez. Sabe-se que o tratamento da hipertensão nas pacientes não gestantes é de fundamental importância para o prognóstico do paciente. Entretanto, nas gestantes existe a necessidade de avaliar o resultado desse tratamento também sobre o concepto (SOUZA et al., 2010).

A interrupção da gravidez é o tratamento definitivo da pré-eclâmpsia. No estágio grave da doença, ou seja, pressão diastólica acima de $110 \mathrm{mmHg}$ e sistólica acima de $160 \mathrm{mmHg}$ persistentemente, cefaléia, distúrbios visuais, deterioração da função renal e síndrome HELLP, requer imediata intervenção (PASCOAL, 2002).

Corrêa et al. (2011) corroboram e acrescentam que em gestações onde a préeclâmpsia se desenvolve precocemente (entra 24 e 32 semanas) e a hipertensão não seja importante, com função renal e hepática estáveis, ausência de sofrimento fetal e/ou distúrbios da coagulação, o parto pode ser postergado até se obter maior maturidade pulmonar fetal, através da corticoterapia.

Admite-se, que a utilização de corticoterapia representa importante terapêutica, não só pela ausência de riscos maternos adicionais, como também pela diminuição de riscos decorrentes da prematuridade fetal. Através de estudos de tratamento da pré-eclâmpsia baseada em evidências, concluiu-se que a corticoterapia esteve associada à significativa redução da mortalidade neonatal, à síndrome do desconforto respiratório do recém-nascido, hemorragia cerebroventricular neonatal e enterocolite necrosante. Não foram identificados efeitos adversos relacionados ao uso do corticóide (NETO et al., 2010).

Estes autores afirmam que não há dados seguros para a conduta conservadora, porém o acréscimo da morbidade neonatal associada à conduta intervencionista 
sugere que a interrupção precoce da gravidez só se justifica se houver risco materno elevado caso a gravidez continua. Acrescenta ainda que a conduta expectante apresente melhor prognóstico perinatal.

Toda gestante na forma grave de pré-eclâmpsia deve ser internada, mantendo monitorização rigorosa com vigilância materna e fetal sendo medicada com sulfato de magnésio, por via endovenosa, para prevenção de convulsões (FERRÃO et al., 2006).

Em estudos, Neto et al. (2010), demonstram que a infusão de sulfato de magnésio se mostrou superior a outras drogas, como diazepam e coquetel lítico. Este procedimento mostrou uma menor taxa de recorrência, redução da morte materna e otimização de resultados fetais.

Porém, independentemente da idade gestacional, a interrupção deve ser considerada na presença de sofrimento fetal ou de sinais de risco materno (PASCOAL, 2002).

Não há evidências de melhora no prognóstico materno e fetal com o tratamento de drogas anti-hipertensivas na hipertensão crônica leve a moderada durante a gestação, mesmo nas pacientes que fazem uso de drogas antes da gravidez (SOUZA et al., 2010).

Recomenda-se o uso de hipotensores somente nos episódios de picos hipertensivos na gestação ou quando se adota conduta conservadora em gestações longe do termo, não estando indicado nas formas leve ou grave da pré-eclampsia. Nas crises hipertensivas, enquanto se prepara a interrupção da gravidez, administra-se drogas anti-hipertensivas para evitar complicações graves, principalmente acidente vascular encefálico. O benefício da medicação anti-hipertensiva nas gestantes com níveis pressóricos menores permanece incerto, indicando o tratamento apenas quando a pressão diastólica é superior a $110 \mathrm{mmHg}$ e sistólica $160 \mathrm{mmHg}$ ou mais (CORRÊA et al., 2011). O objetivo da terapia anti-hipertensiva é de manter a PAS entre $140 \mathrm{mmHg}$ e $155 \mathrm{mmHg}$, e a diastólica entre 90 e 105 mmHg (NETO et al., 2010).

Os principais medicamentos utilizados por via oral são a-metildopa, ß-bloqueador (propranolol) e bloqueadores dos canais de cálcio (nifedipina e nicardipina), enquanto nas emergências hipertensivas são utilizadas medicações intravenosas como hidralazina, nitroglicerina e nitroprussiato de sódio (SASS, 2007).

Nas emergências hipertensivas são utilizadas medicações intravenosas. A hidralazina é o medicamento de escolha, na dose inicial de $5 \mathrm{mg}$ pela via endovenosa, seguida de mais 5 a $10 \mathrm{mg}$ a cada 20 a 30 minutos. A nifedipina, administrada por via oral na dose de $10 \mathrm{mg}$, repetida a cada 30 minutos até se reduzir a pressão diastólica a níveis inferiores a $110 \mathrm{mmHg}$ (CORRÊA et al., 2011).

Em estudo realizado por Ferrão et al. (2006) foi possível concluir que poucos foram os avanços adquiridos em relação à prevenção das intecorrências perinatais, mesmo com a terapia anti-hipertensiva imposta a gestante durante seu acompanhamento perinatal, contudo, este foi de fundamental importância para saúde materna. 


\section{Considerações finais}

Com base nesses achados, considera-se primordial uma assistência pré-natal de qualidade, em que as gestantes de risco sejam identificadas desde a anamnese até o exame clínico. Caso haja necessidade, devem-se realizar intervenções para minimizar as complicações das SHG para mãe e feto.

Recomendam-se, ainda, futuras investigações que deverão contemplar outras variáveis e a realização de estudos prospectivos que permitirão a coleta de informações com maior precisão sobre os potenciais fatores de risco para as SHG.

\section{Referências}

ACOG (American College of Obstetricians and Gynecologists). Committee on Practice Bulletins-Obstetrics. Diagnosis and management of preeclampsia and eclampsia. Int. J. Gynaecol. Obstet. v.77, n.1, p.67-75, 2002.

ACOG (American College of Obstetricians and Gynecologists). Committee on Practice Bulletins-Obstetrics. Chronic hypertension in pregnancy. Obstet. Gynecol. v.98, n.1, suppl 177-85, 2001.

ANGONESI, J.; POLATO, A. Doença Hipertensiva Específica da Gestação (DHEG), incidência à evolução para a Síndrome de HELLP. Rev. Bras. Anal. Clin. v.39, p.243-245, 2007.

ASSIS, T.R.; VIANA, F.P.; RASSI, S. Fatores de risco para hipertensão na gravidez. Arq. Bras. Cardiol. v.91, n.1, p.11-17, 2008.

BUCHBINDER, A.; SIBAI, B.M.; CARITIS, S.; MACPHERSON, C.; HAUTH， J.; LINDHEIMER, M.D.; KLEBANOFF, M.; et al. Adverse perinatal outcome are significantly higher in severe gestational hypertension than in mild preeclampsia. Am. J. Obstet. Gynecol. v.186, n.1, p.66-71, 2002.

CARVALHO, M.A.B.; MELO, V.H.; ZIMMERMMANN, J.B. Resultados perinatais de gestantes com Síndrome Hipertensiva da Santa Casa de Misericórdia de Barbacena, Minas Gerais. Rev. Med. Minas Gerais. v.18, n.4, p.260-266, 2008.

CAVALLI, R.C.; SANDRIM, V.C.; SANTOS, J.E.T.; DUARTE, G. Predição de préeclâmpsia. Rev. Bras. Ginecol. Obstet. v.31, n.1, p.1-4, 2009.

CORRÊA, M.D.; JÚNIOR CORRÊA, M.D.; AGUIAR, R.A.L.P.; MELO, V.H. Noções Práticas de Obstetrícia. Ed. COOPMED, 14a ed. Belo Horizonte: 2011.

COSTA, H.L.F.F, COSTA, C.F.F, COSTA, L.O.B.F. Idade materna como fator de risco para a hipertensão induzida pela gravidez: análise multivariada. Rev. Bras. Gineco. Obstet. v.25, n.9, p.631-635, 2003.

DUSSE, L.M.S.A.; VIEIRA, L.M.; CARVALHO, M.G. Revisão sobre alterações hemostáticas na doença hipertensiva específica da gravidez. J. Bras. Patol. Med. v.37, n.4, p.267-272, 2001. 
FERRÃO, M.H.L.; PEREIRA, A.C.L.; GERSGORIN, H.C.T.S.; PAULA, T.A.A.; CORREAA, R.R.M.; CASTRO, E.C.C. Efetividade do Tratamento de Gestantes Hipertensas. Rev. Assoc. Med. Bras. v.52, n.6, p.390-394, 2006.

GANEM, E.M.; CASTIGLIA, Y.M.M. Anestesia na Pré-Eclâmpsia. Rev. Bras. Anestesiol. v.52, n.4, p.481-49, 2002.

GONÇALVES, R.; FERNANDES, R.A.Q.; SOBRAL, D.H. Prevalência da Doença Hipertensiva Específica da Gestação em hospital público de São Paulo. Rev. Bras. Enferm. v.58, n.1, p.61-4, 2005.

HERCULANO, M.M.S. Avaliação da assistência pré-natal de mulheres com síndrome hipertensiva gestacional. [Dissertação]. Universidade Federal do Ceará. Fortaleza. 2010.

KHAN, K.S.; WOJDYLA, D.; SAY, L.; GULMEZOGLU, A.M.; VAN LOOK, P.F.A. WHO analysis of causes of maternal death: a systematic review. Lancet. v.367, n.9516, p.1066-74., 2006.

LAURENTI, R.; JORGE, M.H.P.M.; GOTLIEB, S.L.D. A mortalidade materna nas capitais brasileiras: algumas características e estimativa de um fator de ajuste. Rev. Bras. Epidemiol. v.7, n.4, p.449-60, 2004.

MORSE, M.L.I.; FONSECA, S.C.; GOTTGTROY, C.L.; WALDMANN, C.S.; GUELLER, E. Morbidade Materna Grave e Near Misses em Hospital de Referência Regional. Rev. Bras. Epidemiol. v.14, n.2, p.310-22, 2011.

MOURA, E.R.F.; OLIVEIRA, C.G.S.; DAMASCENO, A.K.C.; PEREIRA, M.M.Q. Fatores de risco para síndrome hipertensiva específica da gestação entre mulheres hospitalizadas com pré-eclâmpsia. Cogitare Enferm. v.15, n.2, p.250-5, 2010.

NETO, N.C.; SOUZA, A.S.R.; AMORIN, M.M.R. Tratamento da pré-eclâmpsia baseada em evidências. Rev. Bras. Ginecol. Obstet. v.32, n.9, p.459-68, 2010.

NHBPEP. National High Blood Pressure Education Program Working Group on High Blood Pressure in Pregnancy. Am J Obstet Gynecol. v.183, n.1, p.S1-22, 2000.

NOVO, J.L.V.G.; GIANINI, R.J. Mortalidade materna por eclampsia. Rev. Bras. Saude Mater. Infant. v.10, n.2, p.209-217, 2010.

OLIVEIRA, C.A.; LINS, C.P.; SÁ, R.A.M.; NETTO, H.C.; BORNIA, R.G.; SILVA, N.R.; et al. Síndromes Hipertensivas da Gestação e Repercussões Perinatais. Rev. Bras. Saúde Matern. Infant. v.6, n.1, p.93-98, 2006.

OLIVEIRA, S.M.J.V.; FREITAS, P. Gestantes com hipertensão arterial: perfil e conduta de enfermagem. Rev. Baiana Enferm. v.17, n.3, p.23-34, 2002. 
ORGANIZAÇÃO MUNDIAL DA SAÚDE (OMS). Relatoria anual de 2005. Disponível em: http: www.portal.saude.gov.br/portal/ arquivos/pdf/relatório_snvs _ac_2ed.pdf. Acesso em 02 dez. 2012.

PASCOAL, I.F. Hipertensão e Gravidez. Rev. Bras. Hipertensão. v.9, n.3, p.256$261,2002$.

PERAÇOLI, J.C.; PARPINELLI, M.A. Síndromes hipertensivas da gestação: identificação de casos graves. Rev Bras Ginecol Obstet. v.27, n.10, p.627-34, 2005.

SANTOS, Z.M.S.A.; OLIVEIRA, F.M.L.; SILVA, M.P.; NASCIMENTO, J.C.; FEITOZA, J.S.; NASCIMENTO, R.O. Fatores de Risco Para a Síndrome Hipertensiva Específica da Gravidez. RBPS. v.22, n.1, p.48-54, 2009.

SILVA, J.C.G.; TIAGO, D.B.; OLIVEIRA, D.F. Síndrome hellp: a importância da investigação laboratorial na pré-eclampsia. Rev. Ciênc. Méd., v.11, n.1, p.61-65, 2012.

SASS, N. Hipertensão Arterial e Nefropatias na Gestação: Diretrizes e Rotinas assistenciais. Universidade Federal de São Paulo. São Paulo: 2a Ed, 2007.

SIBAI, B.M.; ABDELLA, T.N.; SPINNATO, J.A.; ANDERSON, G.D. Eclampsia. V. The incidence of nonpreventable eclampsia. Am. J. Obstet. Gynecol. v.154, n.3, p.581-586, 1986.

SOUZA, A.R.; AMORIM, M.R.; COSTA, A.A.R.; NETO, C.N. Tratamento antihipertensivo na gravidez. Acta Med Port. v.23, n.1, p.77-084, 2010.

Recebido em: 25/02/2013

Aceito em: 25/06/2013 